\title{
Use of a common software system for collaborative clinical research on attentional processes in schizophrenia and ADHD
}

\author{
JAMES M. SWANSON \\ University of California, Irvine, California \\ KEITH NUECHTERLEIN and ROBERT ASARNOW \\ University of California, Los Angeles, California \\ ERIC TAYLOR \\ Institute for Psychiatry, London, England \\ ROBERT HUNT, BENNET SHAYWITZ, and SALLY SHAYWITZ \\ Yale University, New Haven, Connecticut \\ STEVEN POTKIN \\ University of California, Irvine, California \\ TERRY EARLY and C. ROBERT CLONINGER \\ Washington University, St. Louis, Missouri \\ and \\ MICHAEL POSNER \\ University of Oregon, Eugene, Oregon
}

\begin{abstract}
MacArthur Network III, consisting of five nodes (locations) and over 100 investigators, was formed in 1983 to conduct collaborative clinical research on risk and protective factors associated with psychiatric disorders. The common measurement of attentional dysfunction associated with two specific disorders (schizophrenia and attention deficit-hyperactivity disorder) was a goal of a subgroup of 11 Network III investigators. In this paper, the experiences with five approaches for implementing common protocols on laboratory computers for collaborative clinical research are described. The reasons for selecting the approach provided by Schneider's (1988) MEL system are presented and discussed.
\end{abstract}

The John D. and Catherine T. MacArthur Foundation has created several networks for fostering collaborative research. In 1983, Network III was created for investigations of risk and protective factors of the major mental disorders: schizophrenia, depression, and antisocial personality disorder. Norman Garmezy, of the University of Minnesota, was the head of Network III, which consisted of five nodes (locations): the University of California (Irvine), the University of California (Los Angeles), Yale University (New Haven), Washington University (St. Louis), and the Institute for Psychiatry (London). Each node had a principal investigator and 10 or more investigators (mostly psychiatrists and psychologists).

One of the main goals of the network was to foster collaborative research, both within each node and across nodes. To encourage cross-node collaboration, an annual meeting was held each summer. At the first annual meeting (in 1984), a group was established to discuss how a standard set of laboratory tests of attention could be de-

Correspondence may be sent to James M. Swanson, Child Development Center, Department of Pediatrics, University of California, 19172 Jamboree, Irvine, CA 92715 veloped for use in clinical research. Attentional dysfunction is recognized as an important topic in research on schizophrenia (Asarnow \& MacCrimmon, 1981; Nuechterlein, 1983; Posner, Early, Reiman, Pardo, \& Dhawan, 1988) and attention deficit-hyperactivity disorder (ADHD) (Shaywitz \& Shaywitz, 1988; Swanson, 1985; Taylor, 1986).

Over the 5 years of Network III, the MacArthur Attentional Battery Group evolved, and, by 1988, it included the individuals who are listed in Table 1 , along with their primary clinical interest, an example task each contributed to the initial attentional battery, and the computer each used in 1983.

The goal of the attentional group was to establish a common set of computer-administered cognitive tests (the MacArthur Attentional Battery) for the assessment of clinical cases at different nodes. The initial step taken to accomplish this goal was to exchange laboratory tests. At this point, a major roadblock was encountered: At each node, not all tests were computerized, and those that were computerized were implemented using different computer hardware or software. This complicated the sharing of 
Table 1

The MacArthur Attentional Battery Group

\begin{tabular}{lllll}
\hline Investigator & Node & Group & \multicolumn{1}{c}{ Initial Task } & \multicolumn{1}{c}{ Computer } \\
\hline Swanson & UCI & ADHD & Memory Scanning RT & Apple II \\
Potkin & UCI & Schiz & Eye Movements & Atari \\
Nuechterlein & UCLA & Schiz & Degraded Stim CPT & Compaq \\
Asarnow & UCLA & Schiz & Span of Apprehension & Compaq \\
Taylor & IOP & ADHD & Playing Card CPT & Commodore \\
Posner & St. Louis & Schiz & Spatial Attention RT & Apple II \\
Early & St. Louis & Schiz & A-W Conflict RT & Apple II \\
Cloninger & St. Louis & Various & Personality, Learning & - \\
Hunt & Yale & ADHD & Medication Response & - \\
Shaywitz & Yale & ADHD & General Assessment & - \\
\hline
\end{tabular}

computer programs and the standardizing of protocols for clinical research.

Different approaches were attempted to overcome this roadblock: (1) A computer (the Apple IIc) and programs written for it were sent from one node (UCI) to another (London) to duplicate laboratory conditions; (2) a common computer (the Compaq Portable) was selected and purchased for each node, and investigators at the UCLA node hired a programmer to write programs specifically for this system, which all investigators would use; (3) programs already written in BASIC (for the Apple II at UCI and for the Commodore at London) were rewritten for general use on IBM-compatible computers; (4) an available software system on the Apple Ile computer, ApplePsych, from the University of Oregon (Osgood, 1984a, 1984b), was used to transfer programs from the St. Louis node to the UCI node; and (5) the MEL software system (Schneider, 1988) was used to rewrite programs or write new programs for use on IBM-compatible systems. Our experiences with these five approaches are described below.

\section{Four Initial Approaches}

Duplicate systems. The simplest approach we tried for achieving identical systems was the one in which an investigator at one node provided the exact computer and programs to another investigator for use at the collaborating node. The Apple IIc system, although somewhat outdated, was inexpensive enough to use in this way. An Apple IIc system, with $128 \mathrm{~K}$, an internal floppy disk, an external floppy disk, and several programs written to run on it (Swanson, 1985) were sent to the London node to accomplish this simple exchange. Minor problems were encountered: The $120-\mathrm{V}$ power supply for the computer and the display had to be replaced to conform to the $240-\mathrm{V}$ current in London. This simple approach to the problem of transferring programs and software did not provide a satisfactory solution to the problems facing the MacArthur Attentional Battery Group. Many of the programs suggested by the Network III investigators required a computer with more memory and a hard disk, so this approach had limited applicability to our overall goals and will not be discussed in this paper.

Common computer and centralized programming. After a review of the availability and cost of hardware in 1984, the Compaq Portable IBM-compatible computer was chosen as the standard for Network III. A Compaq system, configured with $640 \mathrm{~K}$ of memory, a $20-\mathrm{Mb}$ hard disk, an AST 6-pak (for joystick and buttonpress input), and a Taxan 720 RBG monitor, was purchased for each node.

A part-time programmer was hired to write programs to duplicate two noncomputerized attentional tasks (Nuechterlein's, 1983, Degraded Stimulus Continuous Performance Test [DS-CPT] and Asarnow \& MacCrimmon's, 1981, Span of Apprehension Test [SPAN]) on this hardware. The programmer elected to use the language PL1. To match the degraded display for the CPT described by Nuechterlein (1983), which was derived from overlapping images from projectors, machine-language code was required. Serious problems were encountered with the IBM display protocols, and to solve these problems required extensive trial-and-error work, spread over a period of 2 years.

At the end of this time, an excellent duplication of the original (noncomputerized) DS-CPT and SPAN was provided for pilot work at UCLA. The next concern of the MacArthur Attentional Battery subgroup was to evaluate and standardize the laboratory tests to ensure a link with an extensive literature that recommended the tests. The DS-CPT and SPAN programs are now in use to collect data on normal and clinical subjects at the UCLA and UCI nodes. After this evaluation and standardization, the program will be made available to other investigators for collaborative research.

After the development of the DS-CPT and SPAN programs, other problems were encountered: (1) Refining and revising the initial version of the program required the services of the same programmer, since it was written in an unusual language (PLl); (2) the program required a specific configuration of the Compaq computer to provide precise timing, so revisions were required to transfer it to the next generation machines (i.e., the Compaq Portable III) with faster processors; and (3) the parttime programmer continued to be difficult to contact, which introduced inordinate and unacceptable delays.

To summarize, delays were encountered in the attempt to use a common computer and a centralized programmer. The initial delay was due to technical problems associated with a complex display, which probably was unavoidable. However, subsequent delays associated with program changes and program maintenance were due to 
nontechnical factors and could have been avoided. In this type of project, nontechnical delays due to interactions with a part-time programmer, who has major time commitments elsewhere, are considered to be typical, but unacceptable occurrences. A permanent, full-time programmer who would consider the maintenance of the program a priority, would likely solve this problem. The budget of the present project was too small to hire a full-time programmer; so, in our case, these unacceptable delays probably were unavoidable.

Translating Apple II to IBM BASIC. At the UCI node, when an attempt was made to translate two programs (memory-display scanning and paired-associate learning) from Apple BASIC to IBM BASIC, similar difficulties and delays were encountered. The Apple II programs were written by one of the investigators and were refined over several years of use. To translate the original programs for use on the IBM-compatible system adopted by the MacArthur Attentional Group, a student programmer was hired to work on this project on a parttime basis. The initial time taken to translate the programs was short, but it took a long time to refine and revise the translations. After translation and correction of simple programming mistakes, the task presented by the IBM programs did not exactly duplicate the task presented by the Apple II programs. Subtle changes in programming were introduced in the translation by a new programmer, and revision by the investigator who had written the original versions was difficult. This discouraged the shift at the UCI node from the proven Apple II versions to the IBM versions. Initial attempts to share the programs after translation were not successful for the same reasons, along with other unanticipated problems (e.g., range differences for inputs from different joysticks).

Thus, a simple translation project became difficult when small changes were required. The process of contacting the programmer, providing the descriptions for revision, and convincing him to complete the job was very timeconsuming and frustrating. The final versions of the IBM programs were not refined to make their use easy. Not even the investigator who wrote the original versions of these programs for the Apple II computer used the IBM translations of these programs.

Use of the ApplePsych system to decrease dependence on programmers. Our inability to make (or our difficulty in making) simple changes without having to consult a programmer and the requirement to depend on a part-time programmer for general program maintenance were identified as major problems in the two examples described above. To overcome these problems, the Network III Attentional Group decided to use the approach described by Osgood (1984a, 1984b), who developed the Oregon ApplePsych System. This system was maintained by a full-time permanent consultant, who could be reached without serious delays. The system was written in Pascal, but did not require programming in that language. Instead, ApplePsych was a higher level language, written specifically for use by cognitive psychologists, that provided standard modules for use in acquiring and storing data from laboratory experiments.

To test the use of this higher level language, an exchange of an ApplePsych program on the Apple II computer was initiated. The program selected for the initial exchange was Posner's (1980) PREPSET program, which specifies spatial locations on a computer screen (boxes in the LVF and RVF), presents valid and invalid cues (a brightness increase of one box 100 to $800 \mathrm{msec}$ before the stimulus), and measures a simple reaction time to a nonverbal stimulus (an asterisk presented in one of the boxes).

The ApplePsych system is public-domain software, and it was obtained from Osgood (1984a, 1984b) at no cost. The specific ApplePsych program (PREPSET) that presented the stimuli and collected the RT data in the Posner et al. (1988) study and the ApplePsych program (COMBSTATS) used to summarize the raw data from the experimental conditions were obtained from Jennifer Sandson, who had used the ApplePsych system in collaborative work with the St. Louis investigators.

Initial problems were encountered when the ApplePsych system was tried. The hardware clock card used in the Apple Ile computer at St. Louis (a California Computer Systems Programmable Timer Module 7440) and incorporated into the PREPSET program used for the Posner et al. (1988) study was different from the clock card in the UCI Apple IIe computer (a Mountain Computer Clock). A telephone consultation with Osgood was obtained on the same day it was sought to discuss this problem. Instructions were given over the telephone to a naive user of the ApplePsych system (but who was familiar with the Apple Ie computer), and the necessary changes in the Pascal code of the ApplePsych system were made to switch clocks. A second hardware problem was encountered: In the PREPSET program used in the Posner et al. (1988) study, a parallel interface card from California Computer Systems was used to obtain a keypress response with millisecond resolution; however, duplicates were no longer available. A new source for a replacement (Suzzane Barnes and Associates) was recommended, and a parallel interface card and keypress panel were ordered. While waiting for the card to be delivered, a change in the ApplePsych program was made to allow the PREPSET program to accept a response from the computer keyboard. The timing of this response was contaminated by the Apple IIe's $60-\mathrm{Hz}$ sampling rate for monitoring the keyboard. This change was recommended by Osgood (1984b) for demonstration purposes and was made without a significant delay, on the basis of instructions given over the telephone. A demonstration program was operating at the UCI node by the end of the day.

The availability of a full-time permanent consultant (in this case, Osgood at the University of Oregon) greatly facilitated the process duplicating programs across nodes. In contrast to the long delays described earlier, the transfer of the ApplePsych system was rapid. Four hours after obtaining the items listed above, an example version 
of the PREPSET program was running at UCI. Less than a month later, after duplicates of the input card and keypress device were obtained, the program was used to collect data on normal subjects (college students) and clinical cases (first-break and chronic schizophrenic subjects) at the UCI node by Swanson and Potkin (1988) for comparison to the data from the St. Louis node (Posner et al., 1988). The replication of the expected effects in the normal subjects (significant main effects of cue and delay, but not of visual field) and slight differences in RT performance on PREPSET between the clinical cases at the UCI and the St. Louis nodes have been discussed elsewhere (Swanson \& Potkin, 1988).

As in most clinical research, it was critical to duplicate the procedures exactly, since effects not duplicated across locations are likely to represent differences in the clinical populations (e.g., schizophrenic subjects with or without positive signs, such as auditory hallucinations, medication status, etc.) and may not represent failures to replicate. This standardization was one of the primary goals of the MacArthur Attentional Group. The ApplePsych system allowed the essential duplication of procedures across the UCI and St. Louis nodes in this collaborative Network III project.

One point to be made is that the use of a programming system, ApplePsych, greatly facilitated the exchange of computer programs and the standardization of protocols across nodes in Network III. The availability of a highlevel software system, which generated error-free code, and a full-time and permanent consultant solved the problems of program changes and program maintenance that we encountered in the two other approaches.

The ApplePsych system had one major drawback: It was written for the Apple Ile computer and depended upon floppy disks. Even in 1985, this represented outdated technology. We wanted a similar system that would run on an IBM-compatible computer, with a hard disk for data storage and management.

A grant was written and funded by Network III to duplicate the ApplePsych system on the IBM-compatible Compaq Portable computer. The development of the CompaqPsych system was to be supervised by Osgood at the University of Oregon, with Suzanne Barnes and Associates providing the programmers.

\section{Initial Use of the MEL System}

Before the project was initiated, the MEL system (Schneider, 1988) was released in a preliminary form. After an initial look at the MEL system, Osgood informed the Network III Attentional Group that the duplication of ApplePsych for use on an IBM-compatible computer did not make sense, since the MEL system accomplished the same purpose. He advised us to try the MEL system.

On the basis of this strong recommendation, one of us (J.S.) called to inquire about the system. The Posner (1980) PREPSET paradigm was chosen as a test case to see if the MEL system could be used effectively to duplicate an ApplePsych program on an IBM-compatible com- puter. Schneider (November, 1987, personal communication) kept data on the time course of his initial use of the MEL system in this example: The telephone contact was made at 3:35 p.m. It took until 4:00 p.m. to find the appropriate Posner article describing the PREPSET task and to read the procedure section. At 4:18 p.m., the program was written in MEL. At 4:43 p.m., the stimuli were typed in, with MEL's "insert" option and a built-in character generator used to construct boxes. At 4:47 p.m., the program was revised. At 5:30 p.m., the demonstration disk and the PREPSET program in MEL was delivered to Federal Express. At 10:30 a.m. the next day, the initial version of the PREPSET task was running at $\mathrm{UCI}$ on the Compaq Portable III.

On the basis of this demonstration, the MacArthur Attentional Group purchased MEL. Two goals were set: (1) to complete the transfer of the PREPSET program from the Apple Ile computer to the family of IBMcompatible computers and (2) to take expert programmers out of the usual investigator-programmer loop during revision and refinement of the program.

Our first attempt to achieve these goals was not without problems. One of us (J.S.), an Apple II aficionado who did not have experience with DOS on the IBM, attempted to learn MEL on his own, using an early unreleased version of the system, with incomplete documentation. This failed. Next, he went to Pittsburgh to learn MEL with some help, after acquiring some experience with DOS 3.2 and the IBM system. This rudimentary instruction, which took 2 days, was provided by undergraduate students who worked as programmers for Schneider at the University of Pittsburgh. At the end of this time, the major forms in the MEL system (experiment, block, trial, insert, and frame) were understood well enough to complete the fillin-the-blanks approach to programming in MEL.

On the basis of this limited knowledge of the MEL system, the PREPSET task was refined by a MacArthur investigator (J.S.) without further help from an expert programmer. Multiple changes were required to match the IBM version to the Apple Ile version of PREPSET. In the MEL system, successive revisions were made and tested in a matter of minutes, in contrast to the previous experience with part-time programmers in which each change required weeks.

To meet the standardization goal set by the MacArthur Attentional Group, the final version of the PREPSET program was used in a pilot study 3 months after the trial with the MEL system was initiated. Data were obtained from a normal control group of college students and a clinical control group of schizophrenic patients at the UCI node to compare to data previously reported by Posner et al. (1988) using the Apple IIe version of PREPSET and to data collected at the UCI node on a duplicate Apple IIe system using ApplePsych. The confirming standardization data were obtained less than 1 year after the MEL project was initiated.

Thus, in a relatively short period of time ( 3 months), the translation process was completed using MEL to re- 
write PREPSET to run an IBM-compatible computer, and data collection for standardization was complete within 1 year. The major problems that introduced long delays, previously encountered when relying on part-time expert programmers using PL1 (at UCLA) or BASIC (at UCI), were avoided. The use of a high-level system by an investigator to obtain error-free computer-generated code and the availability of full-time permanent consultation by telephone allowed us to accelerate the transfer of laboratory tasks across nodes for collaborative clinical research.

After testing MEL (as described above), the MEL system was adopted for use in future development of the MacArthur Attentional Battery. To facilitate this, a halfday short course was developed to instruct MacArthur investigators in the use of the MEL language. In this course, a 2-h lecture-demonstration was developed to explain the basic concepts, including (1) how to use the MEL experiment form to name independent and dependent variables, (2) how to use the trial form to specify the levels of the independent variables, (3) how to use the frame form to specify the sequence of events on the computer screen, and (4) how to use the insert form to specify a stimulus sequence and trial specific information to the other forms. This was followed by a 2-h lab, in which MacArthur investigators (not programmers working for the investigators) used the MEL system to program and run a preliminary version of a new task.

This short course has been presented four times. The first was for the St. Louis node, when one of us (T.E.), using MEL, duplicated the ApplePsych version of the Posner et al. (1988) Arrow-Word conflict RT task. The course was repeated a year later at St. Louis for a research group working with another one of us (R.C.) to develop a new learning test to evaluate laboratory performance on an approach and avoidance learning task, which would relate to personality dimensions derived from rating scales (Cloninger, 1987). The MEL short course was presented to a group (that included naive and sophisticated programmers) at the University of Oregon, and a rudimentary version of a new word-recognition RT task was produced at the end of the session. At UCLA, a short course was presented to a group of investigators who wrote an initial version of the Span of Apprehension task using MEL.

\section{Summary}

In summary, a group of investigators from the MacArthur Network III on risk and protective factors of the major mental disorders have adopted the MEL system (Schneider, 1988) as a way of implementing a common set of attentional tasks on IBM-compatible computers for use in collaborative clinical research across five locations (nodes) in a research network. The goal was to use a common software and to decrease the dependence required by investigators on expert programmers, which was identified as the major source of delay in previous attempts to develop a collaborative attentional battery.

In a period of 3 months, a successful translation of a complicated task (PREPSET) was accomplished. This type of progress had required 2 years in our previous work, using standard programming languages and parttime programmers.

The next step is to complete the standardization of the attentional battery on normal subjects and clinical cases and to make comparisons across nodes in the MacArthur Network III. When this development work is completed, the MacArthur Attentional Battery will be made available to others for use in collaborative clinical research.

\section{REFERENCES}

Asarnow, R. F., \& MacCrimmon, D. J. (1981). Span of apprehension deficits in the post-recovery stages of schizophrenia: Replication and extension. Archives of General Psychiatry, 38, 1002-1011.

Cloninger, C. R. (1987). A systematic method for clinical description and classification of personality variants. Archives of General Psychiatry, 44, 573-588.

Nuechterlein, K. H. (1983). Signal detection in vigilance tasks and behavioral attributes among offspring of schizophrenic mothers and among hyperactive children. Journal of Abnormal Psychology, 92, 4-83.

OsGood, G. (1984a). Hardware implementation and utility manual (Tech. Rep. No. 7). Eugene, OR: University of Oregon, Department of Psychology, Cognitive Lab.

OsGOOD, G. (1984b). Software program development guide (Tech. Rep. No. 8). Eugene, OR: University of Oregon, Department of Psychology, Cognitive Lab.

PosNer, M. I. (1980). Orienting of attention. Quarterly Journal of Experimental Psychology, 32, 3-25.

Posner, M. I., Early, T. S., Reiman, E., Pardo, P. J., \& DhaWAN, M. (1988). Asymmetries in hemispheric control of attention in schizophrenia. Archives of General Psychiatry, 45, 814-821.

SCHNEIDER, W. (1988). Micro Experimental Laboratory: An integrated system for IBM PC compatibles. Behavior Research Methods, Instruments, \& Computers, 20, 206-217.

ShaywTtz, S. E., Shaywitz, B. E. (1988). Attention deficit disorder: Current perspectives. In J. F. Kavanagh \& T. J. Truss (Eds.), Leaming disabilities: Proceedings of the National Conference (pp. 369523). Parktown, MD: York Press.

Swanson, J. M. (1985). Measures of cognitive functioning appropriate for use in pediatric psychopharmacological research studies. Psychopharmacological Bulletin, 21, 887-890.

Swanson, J. M., \& PotkIn, S. G. (1988). Covert and overt direction of attention in schizophrenia. In M. I. Posner (Ed.), The role of attention in normal development and psychopathology (Tech. Rep. No. 88-3, pp. 12-13). Eugene, OR: University of Oregon, Center for Study of Emotion.

TAYLoR, E. A. (1986). Attention deficit. In E. A. Taylor (Ed.), The overactive child (pp. 73-106). Philadelphia: Lippincott. 